\title{
DESIGN OF LOW COST CAPACITANCE SENSOR TO MEASURE SOIL MOISTURE AND ITS PERFORMANCE CHARACTERISTICS FOR APPLICATION IN AGRICULTURAL FIELDS
}

\author{
S.Khaja Syed Shahul Hameed', A.K.Agarwala ${ }^{2}$ \\ ${ }^{1}$ Instrument Design and Development Centre, Indian Institute of Technology, Delhi. \\ Email: 1iitd2005@gmail.com
}

\begin{abstract}
Soil moisture measurements are vital for the irrigation scheduling as it can help in providing adequate quantity of requisite water to the plants for their photosynthesis and survival needs. Ultimately all living beings such as humans, animals feed on agricultural crop produce for their feeding and energy requirements and as such the plants are the vital backbone of the existence of life on earth. As its certain that no plants can survive without water the principal source through which nutrients, fertilizer, minerals are in dissolved form reach to the plants through their roots and hence soil moisture measurement is an essential part and the modern technology should enable the agricultural sector to optimize their water usage otherwise the continual withdrawal of water improperly has led to fall in water table at many places leading to drought prone situation. Also most of the farmers are mostly illiterate and their affordability to have instruments is limited. Its found that the available soil moisture sensors are rather expensive and not farmers friendly. Hence it is proposed in this paper to design a low cost capacitance sensor which is both easy to operate by illiterate person and within the reach of poor Indian farmer and their results are presented herewith.
\end{abstract}

Key words: Agricultural sensor, capacitance sensor, irrigation scheduling, low cost sensor, soil moisture sensor

\section{INTRODUCTION}

There are large number of methods available to measure the soil moisture such as acoustic, axle sensor, capacitive sensor, eddy current position sensor, gravimetric, electrical, electrochemical, electromagnetic, gamma ray attenuation, GIS, granular matrix, inductive sensor, infrared, ISFET sensor, laser sensor, magnetic sensor, magnetic encoders, magnetic float sensor, mechanical, microwave, neutron scattering, optical, photoelectric sensor, piezosensor, pneumatic, psychrometer, radiometric, reed sensor, remote sensing and satellite based, smart sensor, soil conductivity, ultrasonic sensor, velocity differential domain, virrib sensor, voltametric sensor and each of these methods have inherent advantages as well as short comings such as high cost, soil specific calibration, site destruction, high skill necessary to calibrate and operate, inaccuracies, reliability, repeatability in field measurements.

In India, the total geographical spread is about 329Mha and about 97Mha is irrigated by ground and surface water. The principal food crops are cereals such as rice, wheat, maize etc,. Predominant of the natives eat rice or wheat or both for feeding needs. The total area under cultivation of rice is about 44 Mha and wheat is 27 Mha with wheat and rice being rotational crops in most parts of India. The average yield of rice is typically $3.26 \mathrm{t} / \mathrm{ha}$ and wheat is $2.4 \mathrm{t} / \mathrm{ha}$. At these yield levels, the farmer is not able to recover the cost incurred apart from the feeding and survival needs of the family. This resulting-in increase in debt as the MSP for his produce is being fixed by the government and the sustenance of his family of 4-6 members on this small acreage of land holdings is becoming highly unviable. Due to excess pumping out of ground water during the last 3-4 decades of green revolution, about 108Mha of land holdings have become drought prone leading to fall in crop yield as well as farmers income resulting in farmers suicides occurring regularly.

\section{(A) Moisture effects on the dielectric properties of soils}

Permittivity is a measure of the polarizability of the medium subjected to time varying electric field and the fraction of soil sample that possesses this polarizability property is that containing the water molecule dipoles. There is a strong relationship between permittivity, phase velocity and moisture content, conductivity and power attenuation and hence predicting soil moisture from permittivity measurements at frequencies of $100 \mathrm{MHz}$

\section{(B)Electrical properties of water in clay and silty soils}

Relative dielectric permittivity values at $30 \mathrm{MHz}-3 \mathrm{GHz}$ is if the cation exchange capacity of a material is low, the water molecules are orderly arranged around the soil particles and the dielectric values of the bound water layer remain almost independent of frequency. If CEC increases the molecular structure of the bound water layers is disturbed and the water molecules more easily follow the changing ac field so that dielectric value is higher.

Dielectric constant is a sensitive measure of the moisture content of the soil and by incorporating soil as a part of dielectric of capacitor and by measuring the dielectric, soil moisture is estimated at $150 \mathrm{MHz}^{[3]}$.

Addition of water increases the cation exchange capacity due the disturbance in molecular structure of bound water layers thereby altering the dielectric 
permittivity and by estimating the change in permittivity, soil moisture can be estimated.

The amount of capillary water and resultant surface tension and air-water interface present is due to soil texture, soil structure, organic matter, gravity. The electrical properties of soils depend upon magnetic susceptibility, electrical conductivity and dielectric permittivity and polarization is the separation of charge in a material when an electric field is applied. The dielectric value is a measure of relative ability of a material to store a charge for a given applied field strength, while dielectric loss is a measure of the proportion of the charge transferred in conduction and stored polarization such as electron polarization, molecular polarization, orientational polarization and interfacial polarization and the relaxation frequency of the relative macroscopic dipoles associated with interfacial polarization is about $27 \mathrm{MHz}$ and hence all measurements have to be made above $30 \mathrm{MHz}^{[3]}$. Water held in pores by surface tension are likely to contribute to the frequency response of the capacitance probe, which depends on the number of water molecules which are free to relax as their dipoles respond to the field reversals.

Often the methods for ground based sensing of soil water content and remote sensing techniques rely on finding the bulk dielectric permittivity, which is in turn affected by factors other than water content such as interfacial polarization (Maxwell-Wagner effect), Phase configuration and EC. These complex interactions were modeled by Maxwell-Wagner-Bruggeman-Hanai formalism on the basis of differential effective medium approximation. The electromagnetic measurements are easier if the soil is nonconductive and if all water molecules in the soil freely rotate as a function of the applied electromagnetic field. This is in sandy soils wetted with deionized water for up to frequencies $17 \mathrm{GHz}$ (relaxation frequency of the free water). However EM measurements in saline soils and in fine textured soil are more often highly complicated as the ionic conductivity and clay water ion interactions may affect the permittivity reading as these processes are generally a function of measurement frequency. So permittivity may be a function of water content as well as frequency. Different frequencies propagate through a different medium at different velocities if the permittivity of the medium changes with different frequencies and such a medium is described as dispersive dielectric medium ${ }^{[7]}$.

Soil variables such as soil texture, EC, temperature and measuring frequency affect the sensitivity of the capacitance sensor and various research had been done to find the optimum measuring frequency and all have given contradictory results so far ${ }^{[1]}$ and found out that measurement frequency of $50 \mathrm{MHz}$ is minimum required to result in stable soil dielectric permittivity values, but ${ }^{[4]}$ found out at least the measurement frequencies must be of above $500 \mathrm{MHz}$ for stable dielectric permittivity values, however ${ }^{[2]}$ concluded that the measurement frequency must be greater than or equal to $100 \mathrm{MHz}$ to minimize Maxwell-Wagner polarization. The use of these three studies focused on different aspects of sensitivity of measurement i.e., temperature, salinity and soil texture thereby concluding with different results. However ${ }^{[5]}$ concluded that a measurement frequency of $70 \mathrm{MHz}$ is most suitable as the sensitivity of capacitance sensor measurements are sensitive to soil variables like soil texture, electrical conductivity and temperature. But overall all these studies prove that higher measurement frequencies are required for accurate soil moisture measurement at the same time minimizing the sensitivity to changes in soil EC and temperature.

The frequency shift capacitance sensor capable of measuring soil moisture over a range of $(0 \%$ to $45 \%$ by volume) with IEEE 1451 compatible format wireless transmission and reception with RF range of 30-100meters for a particular specific impedance can be used to estimate the water content. Pairs of electrodes are used to measure the water temperature increases the water conductivity resulting in large errors due to skin and proximity effects and hence small sized electrode pairs reduces those undesirable effects to certain extent ${ }^{[9]}$. Rod shaped electrodes were used to measure electrically water content in natural and artificial soils as the capacitive component of soil impedance is a good measure of the water content. The measurement of dielectric soil properties with longer rod shaped electrodes and the electronic components share larger errors during the high water conductivity. At least $10 \mathrm{MHz}$ measurement frequency is necessary to measure the imaginary admittance component and $20 \mathrm{MHz}$ measuring frequency necessary to avoid polarization effect, however at high frequencies skin effect takes place, so to avoid these considerable effects even at high conductivities small sized pair of electrodes are effective.

Fringing capacitance based soil moisture sensor to find the increase in dielectric constant of a soil water mixture with change in water content. The capacitance of root/soil system changes when the contact surface area between roots and the soil increases with growth ${ }^{[6]}$. Usually capacitance measurements are carried out using a pair of electrodes but the short coming of the electrode pair measurement technique is that the system short circuits in water continuous dispersions and hence a new technique is presented which is based on the measurement of capacitance by utilizing the effect the dielectric constant has on the frequency of an oscillator and its observed that while the presence of gas adjacent to the capacitor plate, no reduction in frequency is noticed but if pure water is present by the side of capacitor plate a much larger reduction in frequency is observed as detailed ${ }^{[10]}$. 
At frequencies lower than $1000 \mathrm{MHz}$, the dielectric constant $E$ of a free water is 80 and for dry soil it is 4 , hence measurement of the dielectric constant offers a means for soil moisture estimation ${ }^{[8]}$. Experiments performed earlier at lower order frequencies of $\mathrm{KHz}$ range found out the importance of interfacial polarization effects in heterogeneous materials such as moist soil. The relaxation frequency of dipoles associated with interfacial polarization is $27 \mathrm{MHz}$.

The soil is being considered as matrix as its supports the plants, provides water and minerals as it's consists of water, mineral particles, organic matter as also living organism. In practice the soil moisture content is not necessarily always a very reliable measure of the availability of the water to the plant. A more useful and reliable concept as defined is soil water potential, the soil water potential is the total amount of work required to move the same amount of pure unbound water the same distance. The soil water potential is composed of three components. The matrix potential, the osmotic potential and the pressure potential and the three components are additive as

$$
\psi=\Psi_{0}+\Psi_{m}+\Psi_{p}
$$

The matrix potential corresponding to permanent wilting is $-1500 \mathrm{~J} / \mathrm{kg}$ and field capacity corresponding to $-10 \mathrm{~J} / \mathrm{kg}$ and the available water is the water retained by the soil between wilting point and field capacity which is normally available to the plants and knowing the soil water content is an essential part of crop water management.

\section{Experimental Set up}

\section{A. Hardware}

The necessity of low cost digital sensor keeping in view the financial distress of the Indian marginal farmers, has been designed in-house using the most commonly available and low cost steel with bare minimum circuitry set-up and being tested for its responses at frequencies from $100 \mathrm{~Hz}$ to $100 \mathrm{MHz}$ under controlled environment for increasing the soil moisture content changes in the soil and found to give a very satisfactory results under laboratory experimental conditions. A sensor has been developed to optimize soil moisture at the required level for different crops. The laboratory experiments held in a controlled environment produced encouraging results and hence there is a substantial potential of savings on electricity as well as it will help in mitigating environmental and climate change apart from optimizing the water usage and increasing the agricultural crop yield in India, which is comparatively lower than many other countries for Rice, Wheat, etc.

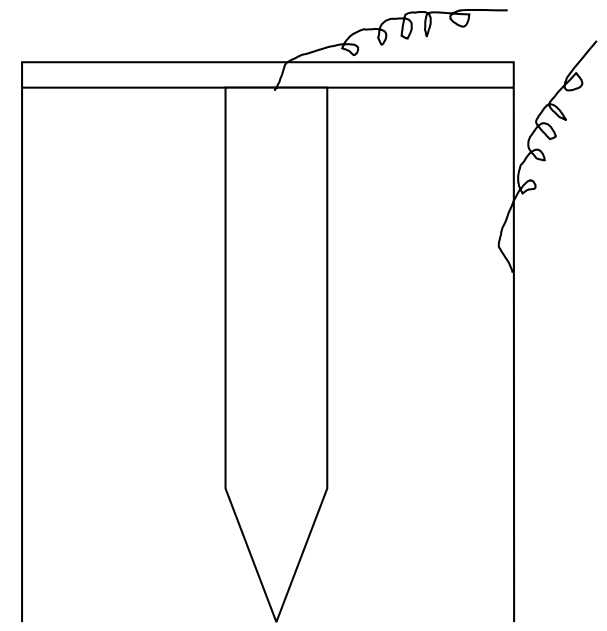

Fig.1.Simple Topology of Capacitance Sensor

The low cost capacitance sensor to measure soil moisture is depicted schematically above has been fabricated using low cost mild steel and used with an impedance measuring system from $100 \mathrm{~Hz}$ to $100 \mathrm{MHz}$ and circuit measurements are being carried out with capacitance in series connection to identify most optimum frequency of triggering the circuit and the above sensor has given highly satisfactory results even at $1 \mathrm{KHz}$ thereby making the circuit simpler and cost effective device suitable for use by Indian farmers and highly viable for field application. The testing with increasing water application at different frequencies has resulted in satisfactory soil moisture measurements. The experiments were performed under laboratory conditions with black soil from sugarcane field from Maharashtra State.

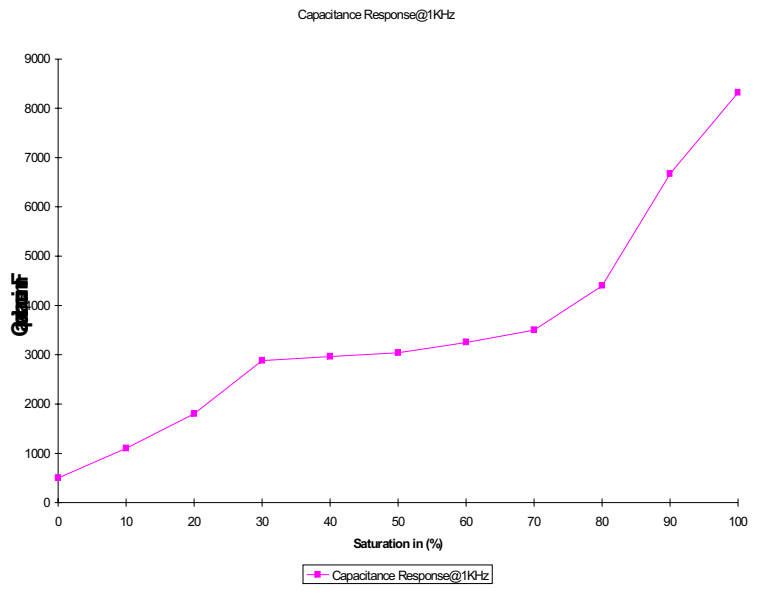

Fig.2.Capacitance Response at $1 \mathrm{KHz}$

\section{B. Results and conclusions}

The scientific observation made from the above sensor response characteristics conclusively establishes a 
fact that it's possible to maintain the soil moisture at a desired level depending on the crop water requirement thereby avoiding full saturation of agricultural fields which results in organic decomposition leading to methane emission and climate change. Using this sensor on large scale in agricultural fields will greatly reduce the GHGs emission from agricultural fields. As its well established fact that crops can survive up to $50 \%$ moisture depletion from their field capacity level and up to permanent wilting point, this low cost capacitance sensor apart from saving the precious ground water resources and electricity will also result in optimizing the crop yield due to better aeration, absorption of nutrients by the plant roots with economic benefit to the nation as a whole.

\section{REFERENCES}

[1] J.E.Campbell., 1990. Dielectric-properties and influence of conductivity in soils at one to $50 \mathrm{MHz}$. Soil Sci. Soc. Am. J. 54 (2), pages 332-341.

[2] Y.Chen, D. Or., 2006. Geometrical factors and interfacial processes affecting complex dielectric permittivity of partially saturated porous media. Water $\mathrm{R}$ e s o u r. R e s. 42 , W06423.doi:10.1029/2005WR00474

[3] T.J.Dean, J.P. Bell and A.J.B.Baty.,1987. Soil moisture measurement by an improved capacitance technique, Part I. Sensor design and performance International Journal of Hydrology, Volume 93, Issues 1-2, Pages 67-78

[4] T.J.Kelleners, D.A. Robinson, P.J. Shouse, J.E. Ayars, T.H. Skaggs., 2005. Frequency dependence of the complex permittivity and its impact on dielectric sensor calibration in soils. Soil Sci.Soc. Am. J. 69 (1), pages 67-76.

[5] F.Kizito,C.S.Campbell, G.S.Campbell ,D.R.Cobos, B.L.Teare, B.Carter and J.W.Hopmans., 2008. Frequency, electrical conductivity and temperature analysis of a low-cost capacitance soil moisture sensor International Journal of Hydrology, Volume 352, Issues 3-4, 15, Pages 367-378 F.
[6] R.E.Miranda, J.B.Yoder, Wilkerson and L.O. Odhiambo.,2005. An autonomous controller for sitespecific management of fixed irrigation systems Computers and Electronics in Agriculture, Volume 48, Issue 3, Pages 183-197 F.R.

[7] V.A.Rinaldi, F.M Francisca.,1999.Impedance analysis of soil dielectric dispersion (1 MHz-1 GHz). J. Geotech. Geoenviron. Eng. 25 (2), pages 111-121.

[8] K.A.Sudduth,S.T.Drummond, and .R.Kitchen.,2001. Accuracy issues in electromagnetic induction sensing of soil electrical conductivity for precision agriculture Computers and Electronics in Agriculture, Volume 31, Issue 3, Pages 239-264

[9] D.Wobschall, and D. Lakshmanan., 2005. Wireless soil moisture sensor based on fringing capacitance Sensors, 2005 IEEE 30 Oct.3 Nov. 2005, Pages 4 pp.

[10] N.Zhang, G. Fan, K.H Lee, G.J Kluitenberg, T.M Loughin., 2004. Simultaneous measurement of soil water content and salinity using a frequencyresponse method. Soil Sci. Soc. Am. J.68, pages1515-1525.

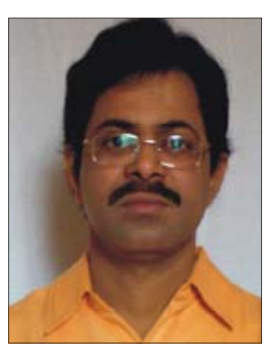

S.Khaja Syed Shahul Hameed obtained B.E Degree (Instrumentation \& Control Engineering)from GCT, Coimbatore and M.E. Degree (Control \& Instrumentation Engg) from College of Engineering ,Guindy and is pursuing research(PhD Programme) at IIT Delhi and has put 2 decades of service in DARE(ICAR), Government of India, CIFRI, Barrackpore and interested in agriculture, and sensors. 